\title{
Personale Autonomie als praktische Autorität
}

\author{
Von CHRISTIAN SEIDEL (Zürich)
}

Der Begriff „personale Autonomie“ ist ein schillernder Begriff. Er wird in unterschiedlichen Zusammenhängen unterschiedlichen Begriffen gegenübergestellt, darunter auch dem Begriff „Autorität“. Bei der Frage nach der Legitimation des Staates etwa setzt eine einflussreiche Begründung für den Anarchismus bei dem vermeintlichen Gegensatz von Autonomie und Autorität an:

„The defining mark of the state is authority, the right to rule. The primary obligation of man is autonomy, the refusal to be ruled. It would seem, then, that there can be no resolution of the conflict between the autonomy of the individual and the putative authority of the state." (Wolff 1970/1998, 18)

Und wenn es um die Erziehung von Kindern geht, so scheinen (kindliche) Autonomie und (elterliche) Autorität ebenfalls in einem gewissen Spannungsverhältnis zu stehen (vgl. Gibbs 1979, 119): Will man Kinder darin unterstützen, autonome Wesen zu werden, so scheint es ratsam, Autorität - Kontrolle, Unterwerfung, Zwang und das Einfordern von Gehorsam und Folgsamkeit - nur sparsam auszuüben.

In diesem Aufsatz möchte ich eine andere Seite des Verhältnisses zwischen den Begriffen „Autonomie“ und „Autorität“ beleuchten. Die Autonomie von Personen kann nämlich, so werde ich argumentieren, auch als eine bestimmte Form von praktischer Autorität verstanden werden; „Autonomie“ ist dann kein Gegenbegriff, sondern ein Unterbegriff zu „Autorität“. Ich möchte zeigen, dass diese Perspektive fruchtbar ist, um etwas über die (Art der) Bedingungen zu erfahren, unter denen eine Person autonom ist.

I.

Die Idee personaler Autonomie spielt in unserem Selbstverständnis, in unserer eigenen Lebensführung, im Umgang mit anderen Menschen und in der Gestaltung politischer Institutionen eine wichtige Rolle. Allerdings ist es nicht leicht, genauer anzugeben, worin die Autonomie einer Person eigentlich besteht oder unter welchen Bedingungen eine Person autonom ist. Offenbar ist „Autonomie“ also ein unscharfer Begriff. Verschiedene philosophische Autonomiekonzeptionen haben es sich zur Aufgabe gemacht, diesen Begriff zu schärfen. Derartige Schärfungen beginnen häufig mit einer „,begrifflichen Annäherung“: Man umschreibt die unscharfe Idee der Autonomie zunächst durch eine weitere, ähnlich unscharfe Idee, an 
welcher sich dann die weitere Untersuchung orientiert. Ein Blick in die gegenwärtige Diskussion zeigt, dass diese erste Annäherung nicht selten mithilfe der Idee der Autorität vollzogen wird:

„[A] utonomy confers normative authority over one's life; the authority to make decisions of practical importance to one's life, for one's own reasons, whatever those reasons might be.“ (Mackenzie 2008, 512)

„[T] o be autonomous is to act within a framework of rules one sets for oneself; that is, it is to have a kind of authority over oneself as well as the power to act on that authority. [...] A theory of autonomy must explain what kind of authority and power is involved." (Oshana 2006, 1 f.)

„When I talk of autonomy it is, in particular, this idea of self-governance that is my direct concern. [...] [I]n self-governance the agent herself directs and governs her practical thought and action. [...] [F]or the agent to direct thinking and acting is for relevant attitudes that guide and control that thinking and action to have authority to speak for the agent - to have agential authority.“ (Bratman 2007, 4 f.)

,[A]utonomous agents, who fully own their wills, act for reasons for which they possess a special authority to speak or answer.“ (Benson 2005b, 102)

In diesen Zitaten kommt explizit die Vorstellung zum Ausdruck, Autonomie sei eine bestimmte Form von Autorität. Diese Vorstellung hat insofern eine Grundlage in unserem Sprachgebrauch, als man eine Reihe von Dingen, die man (spezifisch) von Autoritäten sagen kann, auch von autonomen Personen sagen kann: Man kann die Autorität einer Person untergraben, angreifen, gefährden, verletzen, (miss)achten, respektieren und anerkennen - genauso wie man die Autonomie einer Person untergraben, angreifen, gefährden, verletzen, (miss)achten, respektieren oder anerkennen kann. Und so wie man die eigene Autorität ausüben, bewahren und behaupten kann, kann man auch seine Autonomie ausüben, bewahren und behaupten. Die expliziten Bekenntnisse in der philosophischen Diskussion und die Ähnlichkeiten im begrifflichen Geflecht, in das die Begriffe „Autonomie“ und „Autorität“ jeweils eingebettet sind, legen die Vermutung nahe, dass Autonomie eine Form von Autorität sein könnte.

Dass diese Vermutung zutrifft, ergibt sich aus der folgenden Überlegung: Für unser Verständnis von Autonomie ist es charakteristisch, dass die Antwort auf die Frage, ob eine Person autonom ist oder nicht, Auswirkungen auf das berechtigte Verhalten gegenüber dieser Person hat. Das heißt, dass wir mit einer autonomen Person anders umgehen sollten als mit einer heteronomen Person: Die Willensbekundung der autonomen Patientin sollte von der Ärztin anders berücksichtigt werden als die Willensbekundung der heteronomen Patientin; um die fremdbestimmte Frau, die von ihrem Mann unterdrückt wird und ein Kopftuch tragen muss, sollten wir uns sorgen (und wir sollten ihr vielleicht die Telefonnummer einer Beratungsstelle geben); und der alternden Frau, die ihr Leben nicht mehr selbstbestimmt führen kann, sollte man helfen, während man der alternden Frau, die noch sehr gut alleine zurechtkommt, seine Hilfe nicht aufdrängen sollte. Abhängig von der Autonomie einer Person verändern sich also offenbar unsere normativen Beziehungen zu dieser Person. Man könnte auch sagen, dass es zu so etwas wie einem ,normativen Umschwung“ kommt. ${ }^{1}$ Dieser normative Umschwung ist unmittelbar in der Idee der Autonomie angelegt, er gehört zur Bedeutung des Begriffs „Autonomie": Wer nicht verstanden hat, dass es einen Unterschied für den berechtigten Umgang

1 Eine ähnliche Terminologie („Umschwung in den ethischen Beziehungen“) findet sich im Zusammenhang mit dem Hirntod bei Stoecker (2010), 21. 
mit Personen macht, ob der Begriff „Autonomie“ auf eine Person zutrifft oder nicht, der hat nicht vollständig verstanden, was es heißt, autonom zu sein. Der Umschwung in den normativen Beziehungen gehört einfach zur Idee personaler Autonomie. ${ }^{2}$

Der ,normative Umschwung“, der sich ergibt, wenn eine Person ihre Autonomie gewinnt oder verliert, lässt sich etwas genauer beschreiben als eine Veränderung in den praktischen Gründen, die für die beteiligten Personen vorliegen: Die Ärztin hat bei der Willensbekundung einer autonomen Person einen Grund, bestimmte Behandlungen zu unterlassen (nämlich jene Behandlungen, die die Patientin nicht an sich durchgeführt bekommen möchte), den sie nicht hat, wenn die Patientin heteronom wäre. Wenn eine Frau autonom zum Islam konvertiert und autonom ein Kopftuch zu tragen beginnt, haben wir Grund, von der Weitergabe der Nummer der Frauenberatung abzusehen und diese Entscheidung zu respektieren - einen Grund, den wir im Fall der heteronomen Frau, die von ihrem Mann unterdrückt und gezwungen wird, den Schleier zu tragen, nicht haben. Und während wir im Fall der alternden Frau, die nicht mehr allein zurechtkommt, unsere Hilfe stets aufs Neue anbieten dürfen, haben wir im Fall der Frau, die noch gut alles allein regeln kann, Grund dazu, unsere Hilfsangebote nicht immerfort zu wiederholen. Wenn eine Person autonom ist, besteht offenbar ein Grund, bestimmte Handlungen zu unterlassen, der nicht besteht, wenn die Person heteronom ist. Und ebenso wie der normative Umschwung gehört auch das Vorliegen dieses praktischen Grundes ganz wesentlich zur Bedeutung des Begriffs „Autonomie“. Denn wer nicht verstanden hat, dass man einen Grund hat, von bestimmten Handlungen abzulassen, wenn eine Person autonom ist (einen Grund, den man nicht hat, wenn die Person heteronom ist), der hat nicht verstanden, mit welchem normativen Umschwung der Autonomiebegriff verbunden ist. Und wer das nicht verstanden hat, der hat die Bedeutung des Begriffs „Autonomie“ nicht vollständig durchschaut. Die Verwendung des Autonomiebegriffs geht also - auf Grund der Bedeutung des Begriffs - einher mit Aussagen über das Vorliegen eines bestimmten praktischen Grundes. Dieser Grund hat einen charakteristischen Gehalt: Er spricht für die Unterlassung von Eingriffen in die Entscheidung oder die Lebensweise derjenigen Person, die autonom ist. Dass sich Bert autonom für das Tattoo entschieden hat, ist für uns beispielsweise ein Grund, uns nicht in diese Sache einzumischen und in seine Entscheidung einzugreifen (ihn also nicht am Zutritt zum Studio zu hindern oder den Tätowierer zu bestechen); Berts Autonomie ist aber kein Grund, ihm Geld zu schenken oder ihn zum Studio zu fahren. Solche Gründe könnten durch Berts Armut oder durch seine Verletzung (ein gebrochenes Bein) gestiftet werden. Der charakteristische Gehalt des autonomiebezogenen Grundes scheint also in der Unterlassung von Eingriffen in die Entscheidung oder Lebensweise einer Person zu liegen.

Nun ist es aber nicht nur so, dass die Autonomie einer Person lediglich ,anzeigt“, dass es einen solchen Grund gibt, Eingriffe zu unterlassen; vielmehr ist es so, dass die Autonomie einer Person selbst der Grund ist, aus dem man bestimmte Dinge tun oder unterlassen sollte. Das sieht man daran, wie der Begriff „Autonomie“ typischerweise in Begründungskontexten funktioniert: Angenommen, wir fordern Bert auf, er solle es unterlassen, Clara zu etwas zu zwingen. Wenn Bert nach einer Begründung fragt, warum er Clara nicht zwingen sollte, dann könnten wir antworten: „weil es Claras Autonomie verletzt“" oder „weil Clara sich autonom für etwas anderes entschieden hat als das, was du ihr aufzwingen willst". Hier ist die Tatsache, dass Zwang die Autonomie einer Person verletzt, beziehungsweise die Tatsache, dass eine bestimmte Entscheidung autonom war, der Grund, aus dem Zwang unterlassen werden sollte: Man sollte es unterlassen, weil die Person autonom ist. Tatsachen über die Autonomie von

2 Das ist vereinbar damit, dass zu dieser Idee noch mehr gehört. 
Personen sind (oder konstituieren) also praktische Gründe für die Unterlassung von Eingriffen. ${ }^{3}$

Um nun zu sehen, dass Autonomie eine Form praktischer Autorität ist, muss man etwas genauer betrachten, wie praktische Autoritäten funktionieren (vgl. dazu Raz 1986, Kap. 2 u. 3). Auch bei einer praktischen Autorität kommt es nämlich zu einer Veränderung in den normativen Beziehungen zwischen Personen. Wenn zum Beispiel meine Nachbarin ihre Ausbildung zur Polizistin abgeschlossen hat, dann erwirbt sie gegenüber mir als Verkehrsteilnehmer eine praktische Autorität, die sie zuvor nicht hatte: Sie kann als Polizistin von mir berechtigterweise verlangen, dass ich mit dem Fahrrad anhalte, wenn sie das sagt. Gegenüber Personen, die uns gegenüber praktische Autorität haben, sollten wir uns also anders verhalten als gegenüber Personen, die uns gegenüber keine praktischen Autorität haben. Ebenso wie bei personaler Autonomie findet auch bei praktischer Autorität so etwas wie ein ,normativer Umschwung" statt. Das ist die erste Parallele zwischen Autonomie und Autorität.

Dass $A$ gegenüber $B$ praktische Autorität hat, heißt zunächst, dass $A$ von $B$ berechtigterweise verlangen darf, dass $B$ etwas Bestimmtes tut oder unterlässt. ${ }^{4}$ Es heißt aber noch mehr: Die Polizistin beansprucht mit ihrer Anweisung „Halten Sie an!“ nämlich eigentlich, dass ich anhalte, weil sie es angewiesen hat. Denn nur wenn der Grund, aus dem ich das Rad anhalte, auch wirklich die Tatsache ist, dass die Polizistin mich dazu aufgefordert hat, kann man auch davon sprechen, dass die Polizistin mir gegenüber ihre Autorität ausgeübt hat. Wenn ich das Rad hingegen anhalte, weil mir gerade das Brot aus dem Korb gefallen ist oder ich nach dem Weg fragen wollte, dann geht meine Handlung nicht auf die Autorität der Polizistin zurück. Dass $A$ gegenüber $B$ praktische Autorität hat, heißt also, genauer betrachtet, dass $A$ von $B$ berechtigterweise verlangen darf, dass $B$ etwas Bestimmtes tut oder nicht tut, weil sie, die Autorität $A$, es verlangt. Und das heißt nichts anderes, als dass die Adressatin der Anweisung einen praktischen Grund hat, das Angewiesene zu tun - einen Grund, der durch die Anweisung der Autorität selbst (durch die Tatsache, dass die Autorität es angewiesen hat) konstituiert wird. Wenn $A$ Autorität gegenüber $B$ hat, dann bedeutet dies also, dass die Anweisung von $A$ für sich genommen ein Grund für $B$ ist, das zu tun, was $A$ angewiesen hat (vgl. Raz 1986, 35). Die Anweisung einer Person ohne praktische Autorität hingegen stellt keinen solchen Grund dar.

Ebenso wie der für personale Autonomie charakteristische normative Umschwung lässt sich also der für praktische Autorität charakteristische normative Umschwung beschreiben als eine Veränderung in den praktischen Gründen. Das ist die zweite Parallele zwischen Autonomie und Autorität. Im Fall der Autorität gilt nämlich:

Wenn $A$ Autorität hat, dann ist die Tatsache, dass $A$ als Autorität $B$ angewiesen hat, $H$ zu tun oder zu unterlassen, ein Grund für $B, H$ zu tun oder zu unterlassen.

Und im Fall der Autonomie gilt nun:

Wenn $A$ autonom ist, dann ist die Tatsache, dass $A$ autonom $H$ tut (beziehungsweise autonom auf Weise $H$ lebt), ein Grund für $B$, Eingriffe in $H$ zu unterlassen.

3 Im Folgenden verwende ich die etwas künstliche Redeweise ,autonomiebezogener Grund“, um abkürzend auf diesen durch Autonomie konstituierten Grund Bezug zu nehmen.

4 Dass $A$ dies berechtigterweise verlangen darf, heißt, dass hier von legitimer Autorität beziehungsweise Autorität de jure die Rede ist. Man kann auch in einem de facto-Sinn von praktischen Autoritäten sprechen; man meint dann, dass $A$ (zum Beispiel durch körperliche Überlegenheit) die Macht hat, von $B$ verlangen zu können, etwas Bestimmtes zu tun oder zu unterlassen; dabei bleibt offen, ob diese Macht legitim ist. In diesem Aufsatz verwende ich den Ausdruck ,praktische Autorität“ stets im de jure-Sinn. 
Wenn eine Person autonom ist, dann haben ihre Handlungen oder ihre Lebensweise für andere Personen also denselben normativen Status wie die Weisungen einer Autorität: Es sind für sich genommen praktische Gründe für andere, bestimmte Dinge zu unterlassen. Damit kann man die Autonomie einer Person als eine bereichsspezifische Form praktischer Autorität auffassen: Während praktische Autorität (im Allgemeinen) darin besteht, von anderen berechtigterweise verlangen zu können, dass sie etwas tun oder unterlassen, weil man (die Autorität) es verlangt, besteht Autonomie darin, von anderen berechtigterweise verlangen zu können, dass sie ganz bestimmte Handlungen - nämlich Eingriffe in die Entscheidungen oder Lebensweise - unterlassen, weil man (die autonome Person) so handelt oder lebt.

Eine dritte Parallele zwischen Autonomie und Autorität ergibt sich daraus, dass die praktischen Gründe dasselbe strukturelle Merkmal teilen. Die Anweisungen einer praktischen Autorität konstituieren nämlich Gründe besonderer Art: Dass ein Grund besteht, der autoritativen Anweisung Folge zu leisten, hängt nicht davon ab, was genau angewiesen wird. Ich hätte auch Grund, die Anweisung der Polizistin zu befolgen, wenn diese lautete: „Halten Sie nicht an, fahren Sie bitte zügig weiter!“ Der Grund, den eine Autorität generiert, ist somit unabhängig vom Inhalt der Anweisung: Egal ob es richtig oder falsch ist, wenn eine legitime praktische Autorität es befiehlt, dann ist dies ein Grund, es zu tun. Man kann daher sagen, dass die Anweisungen einer praktischen Autorität für sich genommen ,inhaltsunabhängige“ praktische Gründe sind, das zu tun, was angewiesen wird (vgl. Hart 1970/1990, 101; Raz 1986, 35). Inhaltsunabhängige Gründe kann man an der Zulässigkeit einer bestimmten Reaktion erkennen, wenn der Adressat des Grundes nach einer Begründung fragt: Wenn $A$ als Autorität $B$ anweist, vom Fahrrad zu steigen, und $B$ fragt, warum sie das tun sollte, dann kann $A$ sagen: „Weil ich es gesagt habe!“ Hier erfolgt keine weitere inhaltliche Rechtfertigung der Anweisung, und sie muss auch nicht erfolgen, eben weil $A$ praktische Autorität hat. Das ist gerade die Pointe an einer legitimen praktischen Autorität: Sie sagt uns, was zu tun ist, ohne uns Rechenschaft schuldig zu sein, warum es zu tun ist. In diesem Sinne beruht die normative Kraft des Grundes, der einer autoritativen Anweisung entspringt, nicht auf dem Inhalt der Anweisung.

Auch bei den Gründen, die durch die Entscheidungen oder Lebensweise einer autonomen Person konstituiert werden, handelt es sich um inhaltsunabhängige Gründe: Dass ein Grund gegen einen Eingriff vorliegt, ist nämlich unabhängig davon, wofür sich die Person entscheidet oder wie sie lebt. Wenn Bert sich autonom dafür entscheidet, sich tätowieren zu lassen, dann haben wir Grund, nicht in seine Entscheidung einzugreifen - und diesen Grund haben wir auch, wenn Bert sich autonom gegen ein Tattoo entschieden hätte. Die Inhaltsunabhängigkeit des autonomiebezogenen Grundes erkennt man auch hier an der Zulässigkeit einer bestimmten Reaktion, wenn nach einer Begründung gefragt wird: Angenommen, Bert hat die Entscheidung, sich tätowieren zu lassen, ganz autonom getroffen und seine Mutter versucht nun, ihn umzustimmen oder ihn am Gang ins Tätowierstudio zu hindern. Wenn Bert ihr nun sagt, sie solle sich nicht einmischen, und die Mutter fragt, warum sie aufhören soll, ihn an der Tätowierung zu hindern, dann kann Bert einfach sagen: „Weil ich es einfach will!“ Wenn Bert tatsächlich autonom ist, dann ist auch hier der Verzicht auf eine inhaltliche Rechtfertigung der Unterlassung legitim. Bert tut nichts anderes, als seine „Autorität“ (genauer: seine Autonomie) ins Spiel zu bringen - so wie $A$ gegenüber $B$ ihre Autorität geltend gemacht hat, als $B$ fragte, warum sie vom Fahrrad steigen solle. So wie die normative Kraft des Grundes, der einer autoritativen Anweisung entspringt, nicht auf dem Inhalt der Anweisung beruht, so beruht also auch die normative Kraft des Grundes, der aus autonomen Handlungen oder autonomen Lebensweisen entspringt, nicht auf dem Inhalt der Entscheidungen oder Lebensweisen. Das ist die dritte Parallele zwischen Autonomie und Autorität. 
Das Argument für die These, Autonomie sei eine Form praktischer Autorität, kann man somit wie folgt zusammenfassen: Es ist charakteristisch für unser Verständnis von Autonomie, dass sich mit der Autonomie einer Person auch die normativen Beziehungen zu ihr verändern. Dieser „,normative Umschwung“ ist genauer betrachtet eine bestimmte Veränderung in den praktischen Gründen. Und diese Veränderung in den praktischen Gründen ist von der gleichen Art wie die Veränderung in den praktischen Gründen, die charakteristischerweise durch eine praktische Autorität in Gang gesetzt werden. Also ist Autonomie eine Form praktischer Autorität.

II.

Man könnte nun einwenden, dass mit der These, Autonomie sei eine bestimmte Form praktischer Autorität, nicht viel gewonnen ist; schließlich ist damit noch gar nichts über die Bedingungen gesagt, unter denen eine Person autonom ist. Dieser Einwand ist zunächst ganz berechtigt. Denn tatsächlich wird die eigentlich interessierende Frage nach den Bedingungen personaler Autonomie lediglich transformiert: Wenn Autonomie eine Form praktischer Autorität ist, dann sind die Bedingungen dafür, dass eine Person autonom ist, einfach die Bedingungen dafür, dass sie diese Form von praktischer Autorität hat - und das heißt, es sind Bedingungen dafür, dass ihre Entscheidungen oder Lebensweise einen inhaltsunabhängigen Grund abgeben, Eingriffe zu unterlassen. Die Frage nach den Bedingungen personaler Autonomie ist somit in Wahrheit eine Frage nach den Bedingungen für das Vorliegen eines bestimmten praktischen Grundes - oder, wie man es auch ausdrücken könnte, es ist die Frage nach der Grundlage einer bestimmten Form praktischer Autorität. Die eigentliche Aufgabe von Konzeptionen, die Bedingungen für die Autonomie von Personen angeben, besteht also darin zu klären, auf welcher Grundlage eine spezifische Form praktischer Autorität beruht.

Richtig ist nun, dass nach wie vor offen ist, was genau diese Grundlage ist. Doch entgegen dem Einwand ist die vorgeschlagene Transformation nicht ohne Vorteil. Denn aus ihr lassen sich (mindestens) drei Adäquatheitskriterien gewinnen, die eine Konzeption personaler Autonomie erfüllen muss: Erstens müssen die Bedingungen personaler Autonomie von der richtigen Art sein, um überhaupt eine Grundlage für das Vorliegen eines praktischen Grundes abgeben zu können. Die Autonomiebedingungen können nicht ausschließlich aus normativ irrelevanten Tatsachen bestehen, weil aus einer Ansammlung von normativ Irrelevantem nichts normativ Relevantes - ein praktischer Grund - entspringen kann. Zweitens müssen die gesuchten Autonomiebedingungen auch so beschaffen sein, dass sie die richtige Kategorie von Grund abgeben. Bei dem Grund, der durch die Autonomie einer Person gestiftet wird, handelt es sich ja um einen inhaltsunabhängigen Grund: Seine normative Kraft beruht nicht auf dem Inhalt der Entscheidung oder Lebensweise. Folglich dürfen Autonomiekonzeptionen das Bestehen des Grundes nicht davon abhängig machen, wofür sich eine Person entscheidet oder wie sie lebt. Drittens schließlich müssen die gesuchten Autonomiebedingungen einen inhaltsunabhängigen Grund mit passendem Gehalt abgeben: Der autonomiebezogene Grund ist ein Grund, nicht in die Entscheidungen oder Lebensweise einer bestimmten Person einzugreifen. Der Grund spricht also für die Unterlassung von Eingriffen in die Belange einer ganz bestimmten Person. Die gesuchten Autonomiebedingungen müssen einen Grund mit diesem Gehalt hervorbringen - und nicht einen Grund, der für etwas ganz anderes spricht.

Wie ich nun zeigen möchte, hat ein Teil der gegenwärtig diskutierten Autonomiekonzeptionen Schwierigkeiten, diesen drei Adäquatheitskriterien zugleich gerecht zu werden. Meine These ist also, dass diese Konzeptionen eine bestimmte Form praktischer Autorität auf die falsche Grundlage stellen und deswegen als Konzeptionen personaler Autonomie scheitern. 


\section{III.}

Die gegenwärtig diskutierten Autonomiekonzeptionen bewegen sich zwischen zwei Polen: Auf der einen Seite konzipieren internalistische Ansätze Autonomie überwiegend als ein „Selbstverhältnis“ (ein Verhältnis, das eine Person zu sich selbst oder zu Teilen von sich selbst einnimmt). Die Bedingungen personaler Autonomie betreffen diesem Verständnis zufolge das „Innenleben“ einer Person, ihre ,innere Welt“: Es sind Aussagen über mentale Zustände (etwa „P hat den Wunsch, dass $x^{“}$ ), über Relationen zwischen mentalen Zuständen (etwa „PS Wunsch, dass $x$, ist stärker als $P$ s Wunsch, dass $y^{“}$ ), über die Vermögen der Person (etwa „P kann rational über ihr Tun nachdenken“) oder über Relationen zwischen diesen Vermögen (etwa „Ps rationales Nachdenken über ihr Tun wirkt sich weniger auf ihr Verhalten aus als ihre Neigungen“). Autonomie wird in internalistischen Ansätzen ausschließlich anhand derartiger interner Bedingungen verstanden. Auf der anderen Seite konzipieren externalistische Ansätze Autonomie vor allem als ein Weltverhältnis (im Sinne einer Beschaffenheit der äußeren Welt). Die Bedingungen personaler Autonomie sind diesem Verständnis zufolge externe Bedingungen - Bedingungen, die nicht im definierten Sinne interne Bedingungen sind und die Umstände der äußeren Welt betreffen. Die Bedingung, dass eine Person $P$ nicht von anderen diskriminiert wird, ist beispielsweise eine externe Bedingung, denn damit wird vor allem etwas über andere Personen gesagt und damit betrifft diese Bedingung nicht allein die mentalen Einstellungen von $P$. Soziale Bedingungen (wie „P lebt frei von Zwängen und Anpassungsdruck“), optionsbasierte Bedingungen (wie „P stehen mehrere wertvolle Optionen offen"), historische Bedingungen (wie „P wurde nicht manipuliert") oder substanzielle Bedingungen (wie „P handelt moralisch richtig“) sind weitere Beispiele für externe Autonomiebedingungen, denn Zwang, Anpassungsdruck, Einschränkungen, Manipulation oder die moralische Qualität einer Handlung spielen sich nicht in der inneren Welt einer Person ab, sondern betreffen die sie umgebenden Umstände (ihre äußere Welt).

Das klassische Beispiel für eine internalistische Autonomiekonzeption ist Harry Frankfurts hierarchisches Modell (Frankfurt 1971/1988). Eine autonome Person zeichnet sich Frankfurt zufolge durch ein besonderes Verhältnis zu ihren eigenen Motiven aus: Sie hat einen handlungswirksamen Wunsch erster Stufe W1 nach der Herbeiführung eines bestimmten Sachverhalts und einen Wunsch zweiter Stufe W2, dass W1 handlungswirksam wird (diesen Wunsch nennt Frankfurt eine Volition zweiter Stufe). Bekanntermaßen ist diese Konzeption dem Regressproblem ausgesetzt (Watson 1975, 218): Volitionen zweiter Stufe sind nämlich einfach Wünsche mit einem besonderen Inhalt. Dass sie einen besonderen Inhalt haben, ändert aber nichts daran, dass sie - ihrer Natur nach - eben Wünsche bleiben. Doch von Wünschen kann man entfremdet sein, sie können einen überkommen und äußerlich sein: Wohl jeder kennt Augenblicke, in denen man rot sieht und am liebsten gegen die Wand treten möchte. In diesen Fällen von „Affekttaten“ gilt eine Person gerade nicht als autonom. Diese Möglichkeit der Entfremdung von den eigenen Wünschen besteht ganz unabhängig davon, welchen Inhalt die Wünsche haben. Also besteht diese Möglichkeit auch für Volitionen zweiter Stufe, welche ja lediglich Wünsche eines bestimmten Inhalts sind. Damit können solche Wünsche nicht mehr hinreichend für personale Autonomie sein. Frankfurt müsste Wünsche dritter Stufe einführen, für die sich das Problem aufs Neue stellen würde und so fort.

Dieses Problem lässt sich vor dem Hintergrund der vorangegangenen Überlegungen auch so verstehen: Nach Frankfurts Auffassung fußt die praktische Autorität autonomer Personen auf einer besonderen Konfiguration von Wünschen. Doch das Phänomen der Entfremdung von den eigenen Wünschen zeigt, dass sich Wünsche dafür nicht eignen: Personen, die von ihren Wünschen erster Stufe entfremdet sind, handeln nicht autonom, wenn diese Motive 
handlungswirksam werden; sie haben in diesen Fällen nicht die praktische Autorität autonomer Personen: Ihre Entscheidung ist für sich genommen kein Grund für andere, Eingriffen in ihre Belange zu unterlassen. Das bedeutet aber, dass ein bloßer Wunsch (erster Stufe) nach der Herbeiführung eines bestimmten Sachverhalts für sich genommen keinen Grund gegen eine Einmischung konstituiert. Und darum ist unklar, weshalb ein Wunsch mit einem anderen Inhalt (eine Volition zweiter Stufe) einen solchen Grund konstituieren sollte. Wenn ein Wunsch keinen Grund gegen Einmischung abgibt (und das tut er nicht), dann geben Wünsche mit einem besonderen Inhalt auch keinen solchen Grund ab. Frankfurts Problem folgt somit einfach daraus, dass Wünsche allein keine Gründe sind, Eingriffe in jemandes Entscheidung oder Lebensweise zu unterlassen. Die für Autonomie charakteristische Form von praktischer Autorität kann man somit nicht in Wünschen gründen.

Ein anderes Beispiel für eine internalistische Autonomiekonzeption ist Gary Watsons platonisches Modell (Watson 1975). Darin wird Autonomie als eine Kongruenz von handlungswirksamen Wünschen und bestimmten Werturteilen expliziert: Die autonome Person hat einen handlungswirksamen Wunsch nach der Herbeiführung eines bestimmten Sachverhalts und urteilt, dass die ausgeführte Handlung die alles in allem betrachtet beste ist. Anders als Frankfurt bemüht Watson somit mentale Zustände verschiedener Art, darum ist er nicht - jedenfalls nicht unmittelbar - vom Regressproblem betroffen. Dafür stellt sich ihm ein anderes Problem: Watsons Modell impliziert nämlich, dass jeder Fall von Willensschwäche stets auch ein Fall von fehlender Autonomie ist. Denn im Fall willensschwachen Handelns tut man etwas, wovon man selbst urteilt, dass es nicht die alles in allem betrachtet beste Handlung ist; man glaubt beispielsweise, dass es am besten wäre, mit dem Rauchen aufzuhören oder weniger Schokolade zu essen, greift aber dennoch zur Zigarette oder Schokolade. In diesen Fällen sind Watsons Autonomiebedingungen nicht erfüllt und folglich müssten willensschwache Personen stets heteronom sein. Doch das sind sie nicht: Wir behandeln den willensschwachen Diätbrecher oder die willensschwache Raucherin einfach nicht wie Personen, denen es in paradigmatischer Weise an Autonomie fehlt: Wir bevormunden sie nicht, wir übernehmen nicht die Regelung ihrer Angelegenheiten, wir halten sie nicht für vermindert zurechnungs- oder schuldfähig und wir bieten ihnen nicht einfach umfassende Unterstützung an, wie wir es bei Kleinkindern, Demenzkranken, im Affekt Handelnden oder der unterdrückten Ehefrau tun. Die (normative) Beziehung zu willensschwachen Personen ist eine andere als die (normative) Beziehung zu heteronomen Personen. Und darum identifiziert das platonische Modell nicht die richtigen Bedingungen für Autonomie.

Die Verwechselung von Willensschwäche und fehlender Autonomie kann man auch als Ausdruck der Tatsache verstehen, dass Watson die für Autonomie charakteristische Form von Autorität auf die falsche Grundlage stellt: Denn wenn ein willensschwacher Diätbrecher zur Schokolade oder eine willensschwache Raucherin zur Zigarette greift, so ist die Tatsache, dass es jeweils ein eigener Entschluss war, immer noch ein Grund für andere, von Eingriffen abzusehen. Wenn man versuchen würde, den Diätbrecher umzustimmen oder der Raucherin die Zigarette aus der Hand zu nehmen, so würden wir dies als Eingriff werten, der zu unterlassen ist. Das heißt, dass auch im Fall der Willensschwäche ein autonomiebezogener Grund vorliegt, Eingriffe zu unterlassen - ein Grund, gegen den der Eingreifende verstößt. Daraus folgt aber, dass die Tatsache, dass jemand gemäß seinem Urteil über die alles in allem betrachtet beste Alternative handelt (Watsons Autonomiebedingungen), nicht die Grundlage dieses Grundes sein kann. Denn im Fall der Willensschwäche besteht diese Tatsache gerade nicht, aber dennoch liegt ein autonomiebezogener Grund für andere vor, nicht in die Entscheidung einzugreifen.

Natürlich ist dies keine abschließende Behandlung internalistischer Konzeptionen, denn es gibt eine Vielzahl weiterer interner Bedingungen, die zur Klärung der Idee personaler Autonomie angeführt werden könnten. Allerdings weist die exemplarische Diskussion auf 
ein Problem hin, das alle internalistischen Ansätze gleichermaßen betrifft: Diese versuchen nämlich, die praktische Autorität autonomer Personen auf der Grundlage der inneren Verfassung einer Person zu erklären. Interne Bedingungen scheinen für diesen Zweck aber nicht geschaffen: Wünsche oder Werturteile geben möglicherweise praktische Gründe für diejenige Person ab, die diese Wünsche beziehungsweise Werturteile hat, aber sie konstituieren keinen Grund für andere, Eingriffe in die Entscheidungen oder Lebensweise einer Person zu unterlassen - sie sind in diesem Zusammenhang also normativ irrelevant und erfüllen das erste oben eingeführte Adäquatheitskriterium nicht. Und es scheint, als gelte das für alle internen Bedingungen: Tatsachen über die innere Verfassung einer Person eignen sich nicht als Bedingungen für das Vorliegen des autonomiebezogenen Grundes beziehungsweise als Grundlage für eine praktische Autorität gegenüber anderen Personen. Sie können vielleicht eine Autorität ,gegenüber sich selbst“ begründen - also erklären, inwiefern Motive, die wir durch ein Werturteil oder ein Volition zweiter Stufe billigen, eine besondere Bedeutung für uns selbst zukommt. Doch wenn es darum geht, die Grundlage der für Autonomie charakteristischen Form von Autorität gegenüber anderen aufzuklären, dann führen internalistische Ansätze die falsche Art von Bedingungen an. Und darum scheitern sie.

Externalistische Ansätze führen demgegenüber Bedingungen anderer Art an, nämlich Umstände ,in der äußeren Welt“. Damit sind Tatsachen gemeint, die nicht ausschließlich von der inneren Verfassung der Person abhängen. Nach Ansicht substanzieller Konzeptionen beispielsweise muss die Entscheidung oder Lebensweise einer autonomen Person den richtigen Gehalt haben: Ein Sklave ist gerade auf Grund dieser Lebensweise nicht autonom; einer Frau, die sich zum Objekt männlicher Begierde macht, fehlt es genau wegen dieser (falschen) Entscheidung an Autonomie; und wer kein geeignetes Mittel für den Zweck, den er erreichen will, wählt, der kann seinen wahren Willen nicht umsetzen, weil er so (nämlich: unvernünftig) handelt. Die leitende Idee ist dabei, dass der Inhalt der Entscheidung oder Lebensweise (das, was die Person tut, respektive die Art, wie sie lebt) gewissen Maßstäben der Richtigkeit genügen muss und diese Maßstäbe nicht vom Innenleben der Person abhängen: Ein Dasein als Sklave, die Objektifizierung der eigenen Person oder das Handeln gegen gute Gründe sind dieser Auffassung nach in gewisser Hinsicht falsch und deswegen mit Autonomie unvereinbar - also ganz unabhängig davon, was die betreffende Person wünscht, denkt, fühlt oder was sonst in ihr vorgeht. Substanzielle Autonomiebedingungen finden sich beispielsweise bei Natalie Stoljar $(2000,109)$, Susan Wolf $(1990,71,117)$ oder in der frühen Position von Paul Benson (1987, 469, 475). Sie sind allerdings dem Konfundierungsproblem ausgesetzt (Benson 2005a, 130-132): Das autonome Leben wird darin gleichbedeutend mit dem moralisch guten, dem vernünftigen oder dem gelungenen Leben. Doch das scheint einfach nicht unserem Verständnis von Autonomie zu entsprechen: Wer seine Kollegin belügt oder als Mafioso Geld eintreibt, macht zwar etwas moralisch falsch, aber wir sprechen beiden nicht unbedingt auch ihre Autonomie ab; die Lügnerin und der Mafioso gleichen weder in ihrem inneren Erleben noch in unseren Reaktionen auf sie dementen, im Affekt handelnden oder unterdrückten Personen. Und dem motorradfahrenden, kettenrauchenden Extrembergsteiger mangelt es vielleicht an Vernunft, aber nicht unbedingt auch an Autonomie.

Das Konfundierungsproblem lässt sich vor dem Hintergrund der These, Autonomie sei eine Form praktischer Autorität, anders deuten: Wenn Autonomie tatsächlich darin bestünde, moralisch oder rational zu sein, dann wäre die Tatsache, dass jemand autonom ist, einfach die Tatsache, dass seine Handlung oder Lebensweise moralisch richtig oder rational ist. Diese Tatsache gibt zwar einen praktischen Grund für andere ab, aber dieser Grund spricht nicht für das, wofür der autonomiebezogene Grund spricht: Es ist ein Grund für andere, genauso zu handeln oder zu leben - schließlich ist die Lebensweise oder Handlung ja moralisch richtig oder vernünftig. 
Doch das ist nicht der Gehalt des Grundes, der aus der Autonomie einer Person hervorgeht (die Unterlassung von Eingriffen). Zudem sind die praktischen Gründe, die aus der Moralität oder Rationalität einer Entscheidung oder Lebensweise folgen, im Unterschied zum autonomiebezogenen Grund nicht inhaltsunabhängig. Denn ob ein praktischer Grund vorliegt, es genauso zu tun, hängt von der Rationalität oder Moralität der fraglichen Handlung ab: Wenn es falsch oder unvernünftig ist, dass Bert sich tätowieren lässt, so bestünde für andere auch kein Grund, es ihm nachzutun. Ein solcher Grund (es Bert nachzutun) läge nur dann vor, wenn Bert auf das Tattoo verzichten würde. Also hängt der praktische Grund in diesem Bild vollständig vom Inhalt der Entscheidung oder Lebensweise ab. Der autonomiebezogene Grund hingegen hängt jedoch, wie erläutert, gerade nicht vom Inhalt von Berts Entscheidung ab. Die Autorität der Moral oder die Autorität der Vernunft generieren somit zwar Gründe, doch diese erfüllen das zweite und dritte Adäquatheitskriterium nicht. Die Grundlage der für Autonomie charakteristischen Form praktischer Autorität kann darum nicht in der Moral oder Vernunft liegen.

Einen anderen externalistischen Ansatz verfolgen optionsbasierte Konzeptionen. Demnach sind diejenigen Umstände in der äußeren Welt, welche über die Autonomie einer Person entscheiden, Tatsachen über die ihr zur Verfügung stehenden Alternativen: Eine autonome Person muss einen adäquaten Spielraum von (wertvollen) Optionen haben (Raz 1986, Kap. 14 u. 15; Oshana 1998, 94). Der Spielraum von Optionen ist nach Raz nur dann adäquat, wenn er alle Lebensbereiche umfasst, vielfältige, aber keine zwingenden und zumindest einige moralisch gute Optionen enthält (Raz 1986, 374-381). All diese Bedingungen sind externe Bedingungen: $\mathrm{Ob}$ die verfügbaren Optionen alle Lebensbereiche betreffen, ob sie vielfältig sind, ob eine Option zwingend oder moralisch gut ist, hängt nicht (jedenfalls nicht ausschließlich) vom Innenleben der Person ab. Diese Auffassung ist mit dem „Problem der inneren Perspektive“ konfrontiert: Erstens lässt sich die Adäquatheit des Handlungsspielraums nur durch Rückgriff auf das Innenleben der Person bestimmen. Wenn man sich zum Beispiel bewusst gegen ein Leben mit eigenen Kindern entscheidet oder in seiner Tätigkeit als Berufspianist aufgeht, so verliert man nicht unbedingt an Autonomie, wenn man erfährt, dass man ohnehin gar keine Kinder haben könnte oder dass man aufgrund mangelnder sonstiger Talente keinen anderen Beruf hätte ergreifen können - obwohl damit jeweils in einem wesentlichen Lebensbereich Optionen wegfallen. Und umgekehrt kann der schier endlose Entfaltungsspielraum, der zum Beispiel einer hochbegabten Abiturientin offen steht, sie auch verunsichern, lähmen, ambivalent machen, innerlich spalten und so ihrer Autonomie im Weg stehen. Wie sich ein Optionenspielraum auf die Autonomie einer konkreten Person auswirkt, scheint somit eher von ihrer inneren Verfassung als von externen Umständen abzuhängen. Zweitens kann eine ganze Reihe von Phänomenen mangelnder Autonomie in diesem Ansatz gar nicht erfasst werden: Innere Zerwürfnisse und Zerrissenheit, Konformismus, mangelndes Selbstvertrauen und Obsessionen sind ,innere Barrieren“ für Autonomie, und sie haben nichts damit zu tun, welcher Handlungsspielraum einem zur Verfügung steht. Diese Schwierigkeiten deuten darauf hin, dass eine optionsbasierte Konzeption der inneren Perspektive der Person (der Bedeutung, die die Optionen und der Spielraum aus Sicht der Person haben) nicht gerecht wird.

Auch dieses Problem lässt sich vor dem Hintergrund der These, dass Autonomie eine Form praktischer Autorität ist, verstehen: Wenn es sich bei der Forderung nach einem adäquaten Optionenspielraum um eine für Autonomie konstitutive Bedingung handeln würde, dann müsste die Tatsache, dass eine Person über einen adäquaten Spielraum wertvoller Optionen verfügt, ein Grund sein, der für die Unterlassung von Eingriffen in die Entscheidungen oder Lebensweise der Person spricht. Doch das ist nicht der Fall: Dass ein Handlungsspielraum adäquat ist oder wertvolle Optionen enthält, spricht dafür, diesen Spielraum nicht einzuschränken. Aber das ist nicht dasselbe, wie für die Unterlassung von Eingriffen zu sprechen: 
Man kann einerseits in eine Entscheidung oder Lebensweise eingreifen, ohne den Spielraum von Optionen zu beschränken (etwa durch unnachgiebige Überzeugungsversuche, durch Untergraben des Selbstvertrauens oder durch eine Erziehung zu Konformität). Und man kann andererseits den Spielraum beschränken, ohne in die Angelegenheiten einer Person einzugreifen (etwa indem man jemandem Optionen nimmt, die er ohnehin nicht gewählt hätte und die somit seine Entscheidungen oder Lebensweise gar nicht berühren).

Die Verhaltensweisen, gegen die der Grund spricht, der aus einem adäquaten Spielraum wertvoller Optionen folgt, sind also andere als die Verhaltensweisen, gegen die der Grund spricht, der aus der Autonomie einer Person folgt. Dass damit die dritte Adäquatheitsbedingung nicht erfüllt ist, liegt gerade darin begründet, dass die optionsbasierte Konzeption die innere Perspektive einer Person vernachlässigt. Denn um diejenigen Fälle zu identifizieren, in denen der durch einen adäquaten Optionenspielraum konstituierte Grund gegen dieselben Handlungen spricht, gegen die der autonomiebezogene Grund spricht, muss man bestimmen, welche Formen der Spielraumbeschränkung zugleich auch Eingriffe in Entscheidungen oder Lebensweisen darstellen. Und das lässt sich nur unter Rückgriff auf die innere Perspektive der Person leisten: Erst wenn man weiß, ob jemandem die verbleibenden Optionen genügen oder die verschlossenen Optionen wichtig sind, ob ihn ein bestimmter Spielraum verunsichert oder überfordert, erst dann kann man wissen, ob eine Einschränkung des Handlungsspielraums überhaupt die jeweilige Lebensweise oder Entscheidung berührt und als Eingriff anzusehen ist. Wenn man aber die Adäquatheit des Handlungsspielraums allein an externen Bedingungen festmacht und auf die innere Perspektive verzichtet, dann kann der Gehalt des Grundes, der aus dem Vorhandensein wertvoller Optionen folgt, nicht der Gehalt des autonomiebezogenen Grundes sein. So gesehen, weist das Problem der inneren Perspektive gerade auf jene Fälle hin, in denen der Gehalt des „optionsbasierten Grundes“ und der Gehalt des autonomiebezogenen Grundes auseinanderfallen. Die Schwierigkeit der optionsbasierten Auffassung ist also nur Ausdruck der Tatsache, dass für die zu erklärende Form praktischer Autorität die falsche Grundlage gewählt wurde: nämlich externe Bedingungen, die nicht berücksichtigen, welche Bedeutung der Optionenspielraum aus Sicht der betroffenen Person (aus der inneren Perspektive) hat.

Auch externalistische Positionen sind damit natürlich nicht erschöpfend behandelt. Allerdings legt die exemplarische Diskussion bereits nahe, dass externalistische Ansätze ein grundsätzliches Problem haben: Sie versuchen, die für Autonomie charakteristische Form praktischer Autorität auf der Grundlage von Umständen in der äußeren Welt zu erklären; diese geben zwar einen Grund für andere ab (und erfüllen damit im Unterschied zu internen Bedingungen das erste Adäquatheitskriterium), doch sie konstituieren Gründe der falschen Art: Sie haben den falschen Gehalt oder sind keine inhaltsunabhängigen Gründe. Das ist kein Zufall, sondern hat damit zu tun, dass externalistische Ansätze die innere Verfassung einer Person nicht berücksichtigen und die praktische Autorität der autonomen Person in gewisser Hinsicht darin zu wurzeln scheint: Die Inhaltsunabhängigkeit des autonomiebezogenen Grundes ist gerade Ausdruck der Vorstellung, dass eine Entscheidung nicht zu respektieren ist, weil sie gut oder richtig ist, sondern einfach weil die Person selbst es so entschieden hat (,weil ich es einfach will!" ); die normative Kraft des autonomiebezogenen Grundes entspringt sozusagen der Person selbst. Und der Gehalt dieses Grundes - dass Eingriffe in die Entscheidung oder Lebensweise einer bestimmten Person zu unterlassen sind - enthält die Idee, dass es so etwas wie ein schützenswertes Inneres der Person („,das Selbst“") gibt. Insofern hängen die Spezifika der für Autonomie charakteristischen Form praktischer Autorität von der ,inneren Beschaffenheit der Person" ab. Und darum verwundert es nicht, wenn externalistische Konzeptionen - die gerade nicht auf die Person und ihre innere Beschaffenheit, sondern auf die Merkmale der sie umgebenden Welt schauen - zwar grundsätzlich eine Form praktischer Autorität gegenüber 
anderen begründen können, aber die spezifischen Eigenheiten der praktischen Autorität autonomer Personen nicht erfassen. Denn die vom Externalismus angeführte Autorität (der Moral, der Vernunft oder eines wertvollen Handlungsspielraums) wurzelt - im Unterschied zu der für Autonomie charakteristischen Form von Autorität - gerade nicht in der Person selbst.

\section{IV.}

Es scheint somit, als zeichneten Internalismus und Externalismus ein unterschiedliches, aber gleichermaßen unangemessenes Bild von der Grundlage, auf der die praktische Autorität autonomer Personen beruht (und deswegen auch ein unangemessenes Bild von der Autonomie von Personen). Und damit ist die Frage nach den Bedingungen personaler Autonomie nach wie vor offen. Ich möchte nun einen Vorschlag entwickeln, der in gewisser Hinsicht „Zwischen“ den Polen von Internalismus und Externalismus angesiedelt ist und Autonomie als eine Interaktion zwischen innerer und äußerer Welt - als ein „Sich-in-Beziehung-zur-Welt-Setzen“ - versteht. Dazu ist es zunächst hilfreich, eine Brücke zur Autonomie von politischen Gemeinschaften (wie der autonomen Region Katalonien oder den Schweizer Kantonen) zu schlagen. In seiner Anwendung auf politische Gemeinschaften ist „Autonomie“ ein Begriff des Vertragsrechts, der die normativen Beziehungen zwischen politischen Akteuren regelt: Er diente einst dazu, einzelnen untergeordneten Stadtstaaten im Rahmen eines übergeordneten Machtbereichs (wie dem Attischen Seebund unter der Führung Athens) eine bestimmte Sphäre der Autorität zu sichern, innerhalb welcher der autonome Stadtstaat allein entscheiden und walten durfte (Ostwald 1982, 7 f.; Bickerman 1958, 326 f.; Lévy 1983, 259 f.). Dass eine Gemeinschaft autonom war (oder ist), gab (oder gibt) anderen Gemeinschaften somit stets einen Grund, nicht in die Belange der autonomen Gemeinschaft - ihre „eigenen Angelegenheiten“ - einzugreifen. Ebenso wie im Fall von Personen macht die Frage der Autonomie also auch bei einer politischen Gemeinschaft einen Unterschied für die normativen Beziehungen zu dieser Gemeinschaft. Diese Autorität einer autonomen politischen Gemeinschaft war (und ist) nicht voraussetzungslos, sondern beruht darauf, dass sie (a) eine gewisse Selbstverwaltung aufgebaut hat, innerhalb derer sie ihre inneren Angelegenheiten selbst regeln kann (Hansen 1995, 26); dass sie (b) - zum Beispiel mittels eines polizeilichen Apparats - die innere Sicherheit gewährleisten, das eigene Territorium verteidigen und als Exekutivgewalt wirken kann (Ostwald 1982, 28-30; Bickerman 1958, 329; Lévy 1983, 268; Hansen 1995, 26); und dass sie (c) innerhalb eines übergeordneten Rahmens (Bundesstaat, Staatenbündnisse etc.) in gemeinschaftlich zu regelnden Angelegenheiten, die sie betreffen, mitreden kann (Hansen 1995, 26; Ostwald 1982, 30). Die Autonomie politischer Gemeinschaften scheint also (unter anderem) in ihrer Mündigkeit (oder Selbstständigkeit), Wehrhaftigkeit und Mitsprache zu bestehen.

Ganz ähnlich ist es nun auch bei Personen. So wie eine autonome politische Gemeinschaft ihre inneren Belange regeln kann, wie sie sich zur Wehr setzen kann, wenn jemand ihren Geboten nicht folgt oder andere Gemeinschaften sie angreifen, und wie sie in gemeinschaftlichen Angelegenheiten mitreden kann, kann man auch von der autonomen Person sagen, dass sie ihre eigenen Angelegenheiten regeln kann, dass sie sich wehren kann, wenn sie von außen oder innen angegriffen wird, und dass sie bei gemeinschaftlich getroffenen Entscheidungen, die sie betreffen, mitreden kann. Das sieht man an unserem Umgang mit Kindern: Kleinkinder erfüllen diese Bedingungen nämlich nicht; sie können ihre Angelegenheiten nicht selbst regeln (und sich bei einem Kindergarten oder einer Schule anmelden), können sich nicht gegen äußere Eingriffe (körperlicher oder mentaler Art) wehren, und sie können nicht mitreden und sich einbringen, wenn es um gemeinschaftliche Dinge geht, die sie 
betreffen (etwa einen Umzug der Familie in eine andere Stadt). Dementsprechend behandeln wir Kleinkinder nicht so, wie wir autonome Personen behandeln: Sie werden bevormundet, getäuscht und konditioniert; Eltern treffen im Namen des Kindes Entscheidungen wie die Wahl eines Kindergartens oder einer Schule und sie nehmen das Kind in Schutz, wenn es von anderen Kindern gehänselt wird. Wenn die Kinder im Verlaufe ihrer Entwicklung aber an Mündigkeit, Wehrhaftigkeit und Mitsprache gewinnen, dann verändert sich auch das (angebrachte) Verhalten ihnen gegenüber: Eltern sollten ihre jugendliche Tochter eben nicht mehr „wie ein Kleinkind“ behandeln und die Anmeldung bei einem Sportverein ohne Rücksprache regeln, die Tochter vor den Hänseleien ihrer Mitschülerin in Schutz nehmen oder ihr die Mitsprache bei einem Umzug der Familie verweigern. In dem Maße, in dem ein Kind die Bedingungen der Mündigkeit, Wehrhaftigkeit und Mitsprache noch nicht erfüllt, gilt es noch nicht als autonom (und wird auch entsprechend behandelt); und in dem Maße, in dem es diese Bedingungen schon erfüllt, gilt das Kind schon als autonom (und wird auch dementsprechend behandelt). Mein Vorschlag lautet also, dass ebenso wie bei politischen Gemeinschaften auch bei Personen der für Autonomie charakteristische normative Umschwung - und damit die für Autonomie charakteristische Form praktischer Autorität - auf Mündigkeit, Wehrhaftigkeit und Mitsprache beruht. Eine Person ist demnach in dem Maße autonom, in dem sie ihre eigenen Angelegenheiten regeln kann, sich gegen fremde Eingriffe zur Wehr setzen kann und in gemeinschaftlichen Angelegenheiten, die sie betreffen, mitreden kann.

Eine wichtige Komponente dieses Vorschlags ist, dass der Ausdruck „kann“ dabei in einem speziellen - nämlich: normativen - Sinne verwendet wird: Oft meinen wir mit „(nicht) können“, dass etwas denkbar oder undenkbar ist; ein Dreieck kann zum Beispiel nicht vier Ecken haben und die Bundeskanzlerin kann den Verteidigungsminister entlassen. Manchmal meinen wir mit „(nicht) können“ aber etwas anderes: dass es nämlich (un)gerechtfertigt wäre, es zu tun oder zu erwarten. Am Ende einer äußerst anstrengenden Zeit, in der eine Doktorandin beispielsweise unter großem Zeitdruck ihre Dissertation fertiggestellt hat, könnte sie so etwas sagen wie „Ich kann nicht mehr weiterarbeiten“ oder „Ich kann diesen Druck nicht mehr aushalten“. Ebenso wie Luther mit seiner Aussage „Ich kann nicht anders“ keine Denknotwendigkeit zum Ausdruck brachte, trifft auch die Doktorandin keine metaphysische Aussage: Es gibt eine mögliche Welt, in der sie weiter arbeitet und dem Druck standhält (ebenso wie man sich vorstellen kann, dass Luther seine Lehren eben doch widerruft). Was die Doktorandin eher meint, ist, dass man von ihr nicht berechtigterweise erwarten darf, dass sie weiterarbeitet oder dass sie dem Druck standhält, und dass andere sie nicht so behandeln dürfen, als könne man das berechtigterweise von ihr erwarten. Die Doktorandin beruft sich auf die in unserer moralischen Praxis fest verankerte Vorstellung, dass es normative Standards dafür gibt, welches Maß an Durchhaltevermögen, Fleiß, starkem Willen, körperlicher und mentaler Belastung man von einer Person zu Recht erwarten und verlangen darf. Mit dem normativen (Nicht-)Können sagen wir also stets etwas darüber, dass man eine Handlung (nicht) berechtigterweise von einer Person erwarten (oder auch einfordern) darf.

Genau dieser normative Sinn von „können“ spielt in den Autonomiebedingungen Mündigkeit, Wehrhaftigkeit und Mitsprache eine Rolle: Dass eine Person ihre eigenen Angelegenheiten regeln, sich gegen fremde Eingriffe wehren und in gemeinschaftlichen Angelegenheiten mitreden kann heißt, dass man jeweils von ihr berechtigterweise erwarten darf, dass sie ihre eigenen Angelegenheiten regelt, sich zur Wehr setzt und in gemeinschaftlichen Angelegenheiten mitredet. Nun muss man zweierlei zeigen: (1) Verschiedene Autonomieund Heteronomieerfahrungen lassen sich unter Rückgriff auf diese Bedingungen beschreiben. (2) Die genannten Bedingungen sind tatsächlich Bedingungen für das Vorliegen des autonomiebezogenen Grundes. 
Zunächst zu (1): Eine Person, die an einer fortgeschrittenen Demenz oder einer schweren Depression leidet, schätzen wir als nicht autonom ein. Das hat etwas mit der Bedingung der Mündigkeit zu tun: Die demente Person kann einen großen Teil ihrer eigenen Angelegenheiten (Haushaltsführung, Körperpflege, finanzielle Vorsorge etc.) dauerhaft nicht mehr regeln; und eine schwer depressive Person kann sich um ihre Belange (berufliche Ziele oder alltägliche Besorgungen) nicht mehr kümmern. Dass dabei ein normativer Sinn von „können“ im Spiel ist, sieht man, wenn man die depressive Person mit einem antriebslosen Faulenzer vergleicht: Er ähnelt der Depressiven darin, dass auch er sich nicht um seine berufliche Zukunft kümmert, nicht den Haushalt führt und keine Besorgungen macht. Im Unterschied zur Depressiven halten wir den antriebslosen Faulenzer jedoch für autonom. Das hat etwas damit zu tun, dass der antriebslose Faulenzer seine eigenen Angelegenheiten regeln kann (er hat nur keine Lust dazu), während die Depressive es nicht kann. Dieser Unterschied ist ein Unterschied in unseren normativen Erwartungen: Nach allem, was wir über das Leid und die Auswirkungen einer Depression wissen, würde man von einer schwer depressiven Person nicht berechtigterweise erwarten oder verlangen, dass sie ihre eigenen Angelegenheiten regelt - es ist eine Krankheit. Von dem antriebslosen Faulenzer hingegen darf man es durchaus berechtigterweise erwarten - Antriebslosigkeit und Faulheit sind keine Krankheiten, sondern motivationale Hemmnisse, deren Überwindung man von einer Person zu Recht verlangen darf. Die Autonomie einer Person hängt somit in Teilen von ihrer (normativ verstandenen) Mündigkeit ab.

Auch die Bedingung der Wehrhaftigkeit spielt bei der Beschreibung von Autonomieerfahrungen eine Rolle: Ein Bankangestellter, der mit einer Schusswaffe zum Öffnen des Tresors gezwungen wird, kann sich gegen den Zwang nicht zur Wehr setzen; ein Heroinsüchtiger kann sich gegen den Wunsch nach Heroin nicht wehren. Beiden Personen fehlt es an Wehrhaftigkeit und deswegen an Autonomie. Gegen welche Einflüsse man sich wehren „kann“ und gegen welche nicht, ist eine Frage unserer normativen Erwartungen, wie man am Unterschied zwischen der Sucht nach Heroin und dem Rauchen von Zigaretten sieht: Typischerweise betrachten wir die Heroinsucht als Verlust von Autonomie, das Rauchen hingegen nicht. Der Unterschied hat damit zu tun, dass wir nach unserem besten Wissen über die Wirkung von Heroin und Nikotin, die Folgen des Entzugs und den Grad der körperlichen Abhängigkeit zu dem Schluss gelangen, dass man von einem Heroinsüchtigen nicht erwarten kann, dass er sich dem Einfluss der Droge widersetzen kann, während man dies vom Raucher durchaus erwarten kann: Das Rauchen haben schon viele Leute erfolgreich aufgegeben, an Heroin hingegen sind viele Leute zu Grunde gegangen; die relativ kurzzeitige Fahrigkeit beim Entzug von Nikotin scheint im Vergleich zu Schüttelfrost, Wahnvorstellungen und Schlimmerem beim Entzug von Heroin von ganz anderer Art zu sein. Entsprechend sind wir der Ansicht, dass man vom Raucher zu Recht durchaus verlangen darf, dass er dem Verlangen nach Nikotin widersteht, während man dies vom Heroin-Abhängigen nicht zu Recht verlangen darf. Auch im Fall sozialer Einflüsse spielen normative Erwartungen eine Rolle: Von dem Bankangestellten, der mit der Schusswaffe zum Öffnen des Tresors gezwungen wird, können wir nicht zu Recht erwarten, dass er sich zur Wehr setzt (schließlich riskiert er damit sein Leben) - von dem Bankangestellten, dem damit ,gedroht“ wird, dass er in Zukunft beim zweiten Vornamen genannt wird, wenn er den Tresor nicht öffnet, können wir es hingegen schon zu Recht erwarten. Entsprechend würden wir nur im ersten, nicht aber im zweiten Fall sagen, dass der Bankangestellte sich nicht zur Wehr setzen konnte. Die Bedingung der Wehrhaftigkeit ist als Bedingung personaler Autonomie somit ebenfalls im normativen Sinn zu verstehen.

Das gilt auch für die Bedingung der Mitsprache: Die unterdrückte Hausfrau ist (unter anderem) deswegen nicht autonom, weil sie in Familienangelegenheiten wie Umzug, finanzielle Vorsorge oder gemeinsamen Anschaffungen nicht mitreden kann. Und dass totalitaristische 
Staaten auch unter der Hinsicht der Autonomie problematisch sind, hat ebenfalls etwas damit zu tun, dass die Bürger bei gemeinschaftlichen Angelegenheiten nicht mitreden können. Auch hier bemisst sich die Frage, ob jemand mitreden „,kann“, an normativen Erwartungen: In Nordkorea werden Menschen stark eingeschüchtert; Kritik am Regime wird im Keim erstickt und hart bestraft. Unter diesen Umständen kann man von einer regimekritischen Bürgerin nicht zu Recht erwarten, dass sie sich in die politische Willensbildung einbringt und ihren Standpunkt vertritt - die drohenden Repressionen sind dafür einfach zu hoch. Entsprechend würde man auch sagen, dass die Menschen in Nordkorea in gemeinschaftlichen Angelegenheiten nicht mitreden können und dass es ihnen insofern an Autonomie fehlt. Wenn nun die drohenden Kosten der Regimekritik nicht mehr ganz so hoch sind, dann verändert sich unsere Einschätzung: Auch kritische Stimmen in der ehemaligen DDR oder dem heutigen Russland wurden und werden verfolgt und in ihren Menschenrechten verletzt. Es ist allerdings nicht ganz klar, ob die Repressionen so hoch sind, dass man von einer Person nicht doch berechtigterweise erwarten darf, dass sie in politischen Angelegenheiten die Stimme erhebt und sich einbringt. Und in dem Maße, in dem das weniger klar ist, ist auch weniger klar, dass die Bürgerinnen und Bürger der ehemaligen DDR und des heutigen Russlands in politischen Dingen nicht mitreden konnten beziehungsweise können und dass es ihnen an Autonomie fehlt.

In einer Reihe von Fällen scheint also der Unterschied im Hinblick auf die Autonomie einer Person beschreibbar zu sein als ein Unterschied in ihrer Mündigkeit, Wehrhaftigkeit oder Mitsprache. Mit Blick auf die These, dass es sich bei Autonomie um eine Form praktischer Autorität handelt, muss aber noch gezeigt werden, dass Mündigkeit, Wehrhaftigkeit und Mitsprache einen inhaltsunabhängigen Grund für andere konstituieren, Eingriffe zu unterlassen. Das ist meines Erachtens auch der Fall: (a) Mündigkeit: Dass eine Person ihre eigenen Angelegenheiten (ihm normativen Sinn) regeln kann, ist ein Grund, ihr die Regelung dieser Angelegenheiten auch tatsächlich zu überlassen. Dass wir es $i h r$ überlassen sollten, heißt gerade, dass wir uns nicht in ihre Angelegenheiten einmischen und für sie ihre Angelegenheiten übernehmen sollten - also einen Eingriff unterlassen sollten (das gilt jedenfalls pro tanto, das heißt solange nicht andere gewichtigere Erwägungen dagegen sprechen). Das sieht man am deutlichsten dann, wenn Kinder erwachsen und selbstständig werden: In dem Maße, in dem man von ihnen erwarten darf, dass sie Dinge, die sie allein betreffen (wie die Wahl ihrer Freizeitbeschäftigungen, finanzielle Angelegenheiten, ihre beruflichen Ziele), eigenständig regeln, sollten sich Eltern aus diesen Angelegenheiten zurückziehen. Man sieht es aber auch bei einem Schlaganfall, nach dem ein Patient zunächst fremde Hilfe beim Toilettengang und der Körperpflege benötigt; wenn er seine Mobilität und Selbstständigkeit zurückgewonnen hat, sollte das Pflegepersonal ihn diese eigenen Angelegenheiten auch wieder selbst erledigen lassen. Die Erfüllung der Bedingung der Mündigkeit konstituiert also einen Grund, bestimmte Eingriffe zu unterlassen. Es ist ein inhaltsunabhängiger Grund, da sein Bestehen nicht davon abhängt, wie die mündige Person ihre eigenen Angelegenheiten regelt: Der Grund, Eingriffe zu unterlassen, besteht für die Eltern unabhängig davon, ob die Jugendliche Automechanikerin oder Postbotin werden will; und für das Pflegepersonal besteht der Grund unabhängig davon, ob der Patient sich nun eher oft oder eher selten duscht.

(b) Wehrhaftigkeit: Menschen, die sich nicht wehren können, betrachten und behandeln wir als schutzbedürftig: Wir bieten Hilfe an, gehen vorsichtiger mit ihnen um oder ergreifen für sie Partei. Diese Dinge sollten wir aber unterlassen, wenn die Personen sich selbst wehren können. Das heißt, die Tatsache, dass sich eine Person gegen fremde Eingriffe (im normativen Sinn) zur Wehr setzen kann, ist ein Grund, es sie auch selbst tun zu lassen und es (pro tanto) zu unterlassen, ihr dabei zur Hilfe zu eilen oder uns auf eine andere Weise einzumischen. Wenn man beispielsweise berechtigterweise erwarten kann, dass Bert sich gegen die Einmi- 
schungen seiner Vorgesetzten in sein Privatleben selbst zur Wehr setzt, dann hat sein Kollege Hans Grund, es zu unterlassen, Bert in dieser Angelegenheit mit seinen Ratschlägen zur Seite zu stehen oder die Vorgesetzte zur Rede zu stellen; Hans würde sich damit nämlich in etwas einmischen, was Bert gut selbst in die Hand nehmen kann. Und sobald man von einem Kind berechtigterweise erwarten darf, dass es sich gegen Verbalattacken anderer Kinder selbst zur Wehr setzt und nicht mehr bei den Eltern Zuflucht sucht, sollten die Eltern nicht mehr die Verteidigung des Kindes übernehmen. Die Erfüllung der Bedingung der Wehrhaftigkeit konstituiert also einen Grund, bestimmte Eingriffe zu unterlassen. Es ist ein inhaltsunabhängiger Grund, weil die Frage, ob der Grund vorliegt oder nicht, nicht davon abhängt, wie sich die Person zur Wehr setzt: Ob Bert eine erfolgreiche Methode wählt und der Vorgesetzten das Zugeständnis abringen kann, sich in Zukunft nicht länger in seine Privatangelegenheiten einzumischen, oder ob Bert von sich aus kündigt, weil er diese Person leid ist, oder ob er die Vorfälle beim Geschäftsführer meldet, ist unerheblich dafür, dass für Hans ein Grund besteht, sich aus der Sache herauszuhalten.

(c) Mitsprache: Dass eine Person in gemeinschaftlichen Angelegenheiten (im normativen Sinn) mitreden kann, ist ein Grund, sie auch tatsächlich für sich alleine sprechen zu lassen. Für die Person zu sprechen, von der man berechtigterweise erwarten darf, dass sie selbst mitredet, für sie das Wort zu ergreifen oder sie zu übergehen, wäre gerade eine Form der Einmischung. Stellen wir uns vor, wie ein Paar darüber nachdenkt, das gemeinsame Kind zur Adoption frei zu geben. Wenn der zuständige Beamte sie fragt, wie sie sich entschieden hätten, und die Frau ihm entgegnete: „Ich will es, und mein Mann will es auch“, so wäre es nicht richtig, wenn der Beamte nicht auch noch einmal den Mann fragen würde: Die Entscheidung, das Kind zur Adoption frei zu geben, betrifft beide Elternteile und muss (zumindest bei ungeteiltem Sorgerecht) von beiden gemeinschaftlich gefällt werden. Unter der Voraussetzung, dass der Mann bei dieser Angelegenheit tatsächlich im normativen Sinne mitreden kann, ist dies für den Beamten auch ein Grund, ihn mitreden zu lassen und ihn entsprechend $\mathrm{zu}$ fragen, ob auch er das Kind zur Adoption freigeben möchte. Die Erfüllung der Bedingung der Mitsprache konstituiert also einen Grund, bestimmte Eingriffe zu unterlassen. Es ist ein inhaltsunabhängiger Grund, weil sein Bestehen unabhängig davon ist, wie die betreffende Person in gemeinschaftlichen Angelegenheiten mitredet und wofür sie sich ausspricht: $\mathrm{Ob}$ der Vater für oder gegen die Adoption ist, ist irrelevant dafür, dass er sich einbringen darf, gehört werden muss und nicht übergangen werden darf.

Alle drei Bedingungen sprechen also dafür, bestimmte Formen von Eingriffen zu unterlassen. Dabei betrifft jede Bedingung eine andere Art von Eingriff (Einmischungen in innere Angelegenheiten, Einmischungen bei der Abwehr von Bedrohungen der inneren Angelegenheiten und Einmischungen bei gemeinschaftlichen Angelegenheiten), doch zusammen decken sie einen Großteil der Eingriffe ab, deren Unterlassung wir als Wahrung der Autonomie einer Person ansehen. Die drei vorgeschlagenen Bedingungen sind also die gesuchten Bedingungen dafür, dass der für Autonomie charakteristische inhaltsunabhängige Grund, Eingriffe zu unterlassen, tatsächlich vorliegt. Sie erfüllen zusammengenommen die in Abschnitt II eingeführten Adäquatheitskriterien: Sie sind jeweils normativ relevant (geben Gründe für andere $a b$ ), sind inhaltsunabhängig (ihre normative Kraft beruht nicht auf dem Inhalt einer Entscheidung oder Lebensweise) und haben den richtigen Gehalt (sprechen also je für die Unterlassung von Eingriffen). Mündigkeit, Wehrhaftigkeit und Mitsprache bilden damit zusammen die Grundlage der praktischen Autorität autonomer Personen. 


\section{V.}

Wie verhält sich dieser Vorschlag zu den verworfenen internalistischen und externalistischen Ansätzen? Sind die Bedingungen der Mündigkeit, Wehrhaftigkeit und Mitsprache interne oder externe Bedingungen? Die Antwort ist: Weder noch, es sind Bedingungen eigener Art. Damit ist zweierlei gemeint: Erstens hängt die Frage, wann die Bedingungen der Mündigkeit, Wehrhaftigkeit und Mitsprache erfüllt sind, jeweils sowohl vom Innenleben einer Person als auch von Umständen in der äußeren Welt ab. Dass wir von einer Person nicht berechtigterweise erwarten dürfen, dass sie ihre eigenen Angelegenheiten regelt, liegt manchmal an ihrer inneren Verfassung (etwa einer psychischen Krankheit), manchmal aber auch an den äußeren Umständen (etwa daran, dass sie gefesselt ist). Dass wir von einer Person nicht berechtigterweise erwarten dürfen, dass sie sich gegen etwas (zum Beispiel die Sucht nach Heroin) oder jemanden (zum Beispiel den Bankräuber) zur Wehr setzt, liegt manchmal an etwas in ihr selbst (Wie leidvoll wäre der Entzug für sie?), manchmal aber auch an der Beschaffenheit des Umfelds (Wie glaubhaft ist die Einschüchterung?). Und dass wir von einer Person nicht berechtigterweise erwarten dürfen, dass sie in gemeinschaftlichen Angelegenheiten mitredet, kann ebenso an ihr selbst liegen (vielleicht hat sie ein vermindertes Selbstwertgefühl und traut sich nicht zu, in einem kompetitiven Umfeld ihre Meinung einzubringen) wie an den äußeren Umständen (die Person muss mit Unterdrückung oder Folter rechnen, wenn sie ihre Meinung vertritt). $\mathrm{Ob}$ also eine Person ihre eigenen Angelegenheiten regeln, sich wehren und mitreden kann, hängt sowohl von ihr selbst (von einem Selbstverhältnis und damit von internen Kriterien) als auch von der äußeren Welt (den Weltverhältnissen und damit von externen Kriterien) ab.

Zweitens ist aber keine der Bedingungen der Mündigkeit, Wehrhaftigkeit und Mitsprache vollständig analysierbar als eine Konjunktion von internen und externen Kriterien. Denn der Zusammenhang zwischen Autonomie und den drei Bedingungen Mündigkeit, Wehrhaftigkeit, Mitsprache ist ein ganz anderer Zusammenhang als derjenige zwischen Mündigkeit, Wehrhaftigkeit, Mitsprache und internen beziehungsweise externen Kriterien: Der erste Zusammenhang ist begrifflicher Art - man kann den Begriff „Autonomie“ nur dort sinnvoll ins Spiel bringen, wo man auch die Begriffe „Mündigkeit“, „Wehrhaftigkeit“ und „Mitsprache" ins Spiel bringen kann, also davon reden kann, dass eine Person ihre eigenen Angelegenheiten regeln, sich gegen fremde Eingriffe zur Wehr setzen und in gemeinschaftlichen Angelegenheiten mitreden kann. Der zweite Zusammenhang hingegen ist normativer Art - die Tatsachen über das Innenleben einer Person und ihre äußere Welt, die für das Vorliegen von Mündigkeit, Wehrhaftigkeit und Mitsprache relevant werden, sind ja Tatsachen, die darüber entscheiden, ob man von einer Person bestimmte Dinge berechtigterweise erwarten darf. Und normative Zusammenhänge sind keine begrifflichen Zusammenhänge. Daher lassen sich die Bedingungen der Mündigkeit, Wehrhaftigkeit und Mitsprache nicht - jedenfalls nicht auf begrifflicher Ebene - auf Aussagen über das Innenleben der Person, nicht auf Aussagen über die äußere Welt und auch nicht auf eine Konjunktion von beidem reduzieren. Die Bedingungen personaler Autonomie sind somit eigener - irreduzibel normativer - Art.

Es ist wichtig zu sehen, inwiefern die Grundlage, auf der die praktische Autorität autonomer Personen diesem Vorschlag nach beruht, speziell ist. Die Autorität autonomer Person wurzelt nämlich weder allein im „Selbst“ noch allein in der äußeren Welt, sondern in einer Interaktion zwischen beidem: Die autonome Person interagiert mit der äußeren Welt, indem sie die Angelegenheiten (die durch den Lauf der Welt sozusagen an sie herangetragen werden) regelt, sich gegen äußere Eingriffe zur Wehr setzt und ihre soziale Umwelt durch Mitsprache mitgestaltet. Damit können die grundsätzlichen Schwierigkeiten der zuvor diskutierten Ansätze umgangen werden: Einerseits wird klar, inwiefern die für Autonomie charakteristische 
Form praktischer Autorität in der Person selbst wurzelt (damit hatten externalistische Ansätze Schwierigkeiten). Denn es ist eine bestimmte Form der Aktivität der Person - ihre Interaktion mit der äußeren Welt -, die die Grundlage der Autorität bildet. Andererseits wird aber auch klar, inwiefern die für Autonomie charakteristische Form praktischer Autorität eine Autorität gegenüber anderen ist (das war die Schwierigkeit internalistischer Ansätze). Denn Mündigkeit, Wehrhaftigkeit und Mitsprache sind, wie aufgezeigt, insofern normativ relevant, als sie anderen die Unterlassung verschiedener Eingriffe abfordern. In diesem Sinne bewegt sich die vorgeschlagene Konzeption von Autonomie ,zwischen“ den Polen von reinem Selbstverhältnis (Internalismus) und reinem Weltverhältnis (Externalismus).

\section{VI.}

Ich habe dafür argumentiert, dass personale Autonomie eine Form praktischer Autorität ist, die ihre Grundlage in der Mündigkeit, Wehrhaftigkeit und Mitsprache einer Person hat. Vor diesem Hintergrund ist der eingangs erwähnte „Gegensatz“ zwischen den Begriffen „Autonomie“" und „Autorität“, der sich beispielsweise bei der Legitimation des Staates oder der Erziehung von Kindern abzeichnet, anders zu deuten - nämlich als eine Spannung zwischen zwei Formen von Autorität: Einerseits hat eine autonome Person $A$ kraft ihrer Autonomie Autorität gegenüber einer anderen Person $B$; andererseits hat diese Person $B$ (kraft ihres Amtes oder ihrer pädagogischen Rolle) Autorität gegenüber $A$. Der „Gegensatz“ zwischen Autonomie und Autorität ist letztlich so etwas wie ein „Kräftemessen“ zwischen zwei verschiedenen praktischen Gründen: Auf der einen Seite folgt aus As Autonomie der autonomiebezogene Grund für $B$, Eingriffe in $A$ s Entscheidung oder Lebensweise zu unterlassen; auf der anderen Seite folgt aus $B$ s Autorität gegenüber $A$ der autoritätsbezogene Grund für $A$, das von $B$ Angewiesene auch zu tun. Und immer dann, wenn sich das, was $B$ anweist, nicht mit der Entscheidung oder Lebensweise von $A$ deckt, stehen die beiden Gründe in einem Konflikt, weil dem einen Grund nachzukommen hieße, dem anderen nicht nachkommen zu können. Der „Gegensatz“ zwischen Autonomie (von Person $A$ ) und Autorität (von Person $B$ ) stellt sich im Lichte der vorgeschlagenen Autonomiekonzeption nicht als ein begrifflicher Gegensatz, sondern eher als eine praktisch-normative Unvereinbarkeit dar: Die Autonomie einer Person steht mit der praktischen Autorität einer anderen Person also deshalb in Konflikt, weil Autonomie selbst eine Form praktischer Autorität ist, die sich mit dem Weisungsanspruch einer weiteren Autorität eben nicht verträgt.*

Dr. des. Christian Seidel, Universität Zürich, Ethik-Zentrum, Zollikerstraße 117, 8008 Zürich, Schweiz

\section{Literatur}

Benson, P. (1987), Freedom and Value, in: Journal of Philosophy, 84 (9), 465-486.

Ders. (2005a), Feminist Intuitions and the Normative Substance of Autonomy, in: Personal Autonomy. New Essays on Personal Autonomy and Its Role in Contemporary Moral Philosophy, hg. v. J. S. Taylor, Cambridge, 124-142.

* Für hilfreiche Diskussionen zu den Gedanken dieses Aufsatzes möchte ich Monika Betzler und Gerhard Ernst herzlich danken. 
Ders. (2005b), Taking Ownership: Authority and Voice in Autonomous Agency, in: Autonomy and the Challenges to Liberalism, hg. v. J. Christman u. J. Anderson, Cambridge, 101-126.

Bickerman, E. J. (1958), Autonomia. Sur un passage de Thucydide (I, 144, 2), in: Revue internationale des droits de l'antiquité, 5 (3érie), 313-344.

Bratman, M. E. (2007), Structures of Agency. Essays, Oxford.

Frankfurt, H. G. (1971/1988), Freedom of the will and the concept of a person, in: ders., The importance of what we care about. Philosophical essays, Cambridge, 11-25.

Gibbs, B. (1979), Autonomy and Authority in Education, in: Journal of Philosophy of Education, 13, $119-132$

Hansen, M. H. (1995), The ,Autonomous City-State'. Ancient Fact or Modern Fiction?, in: Studies in the Ancient Greek polis, hg. v. M. H. Hansen u. K. Raaflaub. Historia Einzelschriften, 95, Stuttgart, $21-43$.

Hart, H. L. A. (1970/1990), Commands and Autoritative Legal Reasons, in: Authority, hg. v. J. Raz, Oxford, 92-114.

Lévy, E. (1983), Autonomie et Èleuthéria au Ve Siècle, in: Revue de Philologie, 57 (2), 249-270.

Mackenzie, C. (2008), Relational Autonomy, Normative Authority and Perfectionism, in: Journal of Social Philosophy, 39 (4), 512-533.

Oshana, M. A. L. (1998), Personal Autonomy and Society, in: Journal of Social Philosophy, 29 (1), $81-102$.

Dies. (2006), Personal Autonomy in Society, Aldershot.

Ostwald, M. (1982), Autonomia: Its Genesis and Early History, American Classical Studies, 11, Chico.

Raz, J. (1986), The Morality of Freedom, Oxford.

Stoecker, R. (2010), Der Hirntod. Ein medizinethisches Problem und seine moralphilosophische Transformation, 2. Aufl., Freiburg im Breisgau.

Stoljar, N. (2000), Autonomy and the Feminist Intuition. In: Relational Autonomy: Feminist Perspectives on Autonomy, Agency, and the Social Self, hg. v. C. Mackenzie u. N. Stoljar, New York, 94-111.

Watson, G. (1975), Free Agency, in: The Journal of Philosophy, 72 (8), 205-220.

Wolf, S. (1990), Freedom within Reason, New York.

Wolff, R. P. (1970/1998), In Defense of Anarchism, Berkeley.

\section{Abstract}

Autonomy and authority are often regarded as opposites. In this paper, I argue that autonomy should be conceived of as a specific form of (practical) authority and that this perspective is useful for identifying the conditions of personal autonomy. I will first highlight some structural analogies in the functioning of the concepts "autonomy" and "authority" and explain the resulting constraints on accounts of personal autonomy. I will then show that the problems of certain internalist and externalist accounts of autonomy are rooted in a false understanding of the foundation on which the authority that is characteristic of autonomy rests. To conclude, I present an account in which this foundation is given by a person's maturity (Mündigkeit), defensiveness (Wehrhaftigkeit) and participation (Mitsprache): Thus, a person is autonomous to the extent that she can cope with her own affairs, can defend herself against external encroachments and can participate in common affairs. 